\title{
Tool Condition Monitoring in Grinding Operation Using Piezoelectric Impedance and Wavelet Transform ${ }^{\dagger}$
}

\author{
Pedro Oliveira Junior 1,*, Paulo Aguiar ${ }^{1}$, Rodrigo Ruzzi ${ }^{2}$, Salvatore Conte ${ }^{3}$, Martin Viera ${ }^{1}$, \\ Felipe Alexandre ${ }^{1}$, Fabricio Baptista ${ }^{1}$, and Cristiano Soares Júnior ${ }^{1}$ \\ 1 Department of Electrical Engineering, São Paulo State University; Av. Eng. Luiz Edmundo Carrijo Coube, \\ 14-01, Bauru 17033-360, Brazil; aguiarpr@unesp.br (P.A.); martin.aulestia@unesp.br (M.V.); \\ felipe.alexandre@unesp.br (F.A.); fabriciogb@feb.unesp.br (F.B.); cristiano.soares@unesp.br (C.S.J.) \\ 2 School of Mechanical Engineering, Federal University of Uberlandia, Av. João de Ávila 2121, \\ Uberlandia 38408-100, Brazil; rodrigo.ruzzi@ufu.br \\ 3 Department of Chemical, Material and Industrial Production Engineering, University of Naples Federico II, \\ Piazzale Tecchio 80, 80125 Naples, Italy; salvatore.conte@unina.it \\ * Correspondence: pedrojunior5@aedu.com \\ + Presented at the 6th International Electronic Conference on Sensors and Applications, 15-30 November \\ 2019; Available online: https://ecsa-6.sciforum.net/.
}

Published: 14 November 2019

\begin{abstract}
The purpose of the present study is to monitor tool condition in a grinding operation through the electromechanical impedance (EMI) using wavelet analysis. To achieve this, a dressing experiment was conducted on an industrial aluminum oxide grinding wheel by fixing a stationary single-point diamond tool. The proposed approach was verified experimentally at various dressing tool conditions. The signals obtained from an EMI data acquisition system, composed of a piezoelectric diaphragm transducer attached to the tool holder, were processed using discrete wavelet transform. The approximation and detail coefficients obtained from wavelet decomposition were used to estimate tool condition using the correlation coefficient deviation metric (CCDM). The results show excellent performance in tool condition monitoring by the proposed technique, which effectively contributes to modern machine tool automation.
\end{abstract}

Keywords: piezoelectric sensors; sensor monitoring; electromechanical impedance; wavelet transform; signal processing; grinding operation

\section{Introduction}

Grinding operations are one of the most critical machining processes for manufacturing components that require high surface quality and precision. The effectiveness of the grinding process is significantly defined by the surface condition of the cutting tool (grinding wheel), and it is the dressing operation that is responsible for producing an appropriate topography for the grinding wheel [1-3]. In this sense, the condition monitoring of the dressing tool plays an essential role within grinding operations [4,5]. To achieve this goal, indirect methods of dressing tool condition monitoring have been addressed, which focused on the classification and estimation of wear in single-point dressers through statistics methods derived from sensor signals, such as acoustic emission (AE) and vibration, followed by machine learning based on artificial neural networks (ANNs) and fuzzy systems [5-8]. 
More recently, the electromechanical impedance (EMI) method has taken place in the field of grinding process monitoring based on lead titanate zirconate (PZT) piezoelectric transducers. Preliminary applications have shown the potential of the EMI method for detecting damage in single point dressing tools, as reported in references $[4,9,10]$. On the other hand, it is well-known that the EMI method has been commonly used for different structural health monitoring (SHM) applications [11-13]. Among the various approaches that have been proposed to improve EMI-based damage diagnosis (machine learning, cognitive systems, numerical modeling), the wavelet transform has been used in a significant amount of research in the field of SHM in recent years.For example, in the study by reference [13], the authors proposed to identify damage using the multilevel wavelet transform. Likewise, in reference by [14], the authors employed the wavelet transform to distinguish different types of structural damage. In the same manner, in reference by [12], the wavelet transform was applied to detect damage in the noise environment.

The use of EMI-based wavelet transform with particular focus to the dressing operation is an approach that has not been reported in the literature, which makes the present work innovative. This way, the purpose of this paper is to monitor the condition of the dressing tool contributing to the grinding process optimization. The proposed approach is validated based on experimental dressing trials conducted on an industrial aluminum oxide grinding wheel by fixing a stationary single-point diamond tool. The impedance measurements were processed by using the discrete wavelet transform. This paper presents a more straightforward methodology for tool condition monitoring in grinding operations that effectively contributes to modern machine tool automation.

\section{Experimental Setup}

To verify the effectiveness of the proposed approach, experimental tests were performed for the dressing operation of conventional aluminum oxide grinding wheel (38A150L6VH), with dimensions of $355.6 \times 25.4 \mathrm{~mm} \times 127 \mathrm{~mm}$, from NORTON manufacturer (Porto Alegre, Brazil), using a tangential surface grinding machine, model RAPH 1055, from Sulmecânica. An industrial single-point dresser of a natural diamond was used for the dressing operation. The experiment was conducted following the approaches reported by references $[4,9,15,16]$. Each trial consisted of single dressing passes (grinding wheel and dresser diamond tip interface). Dressing speed ( $v f d$ ) was kept constant at 3.45 $\mathrm{mm} / \mathrm{s}$, with a dressing depth $\left(a_{d}\right)$ of $40 \mu \mathrm{m}$, and without the use of cutting fluid to provide more pronounced wear. Diamond wear measurements of the dressing tool were performed at 100-pass intervals throughout the test using photographs taken from a DIGIMICRO 2.0 digital microscope, from DNT (Fremont, CA, USA), with 20x magnifications, for comparative purpose. The diamond's worn area was measured using computer-aided design (CAD) software. Figure 1 shows the experimental setup and the DAQ system used in the present study, based on the approach reported in [9].

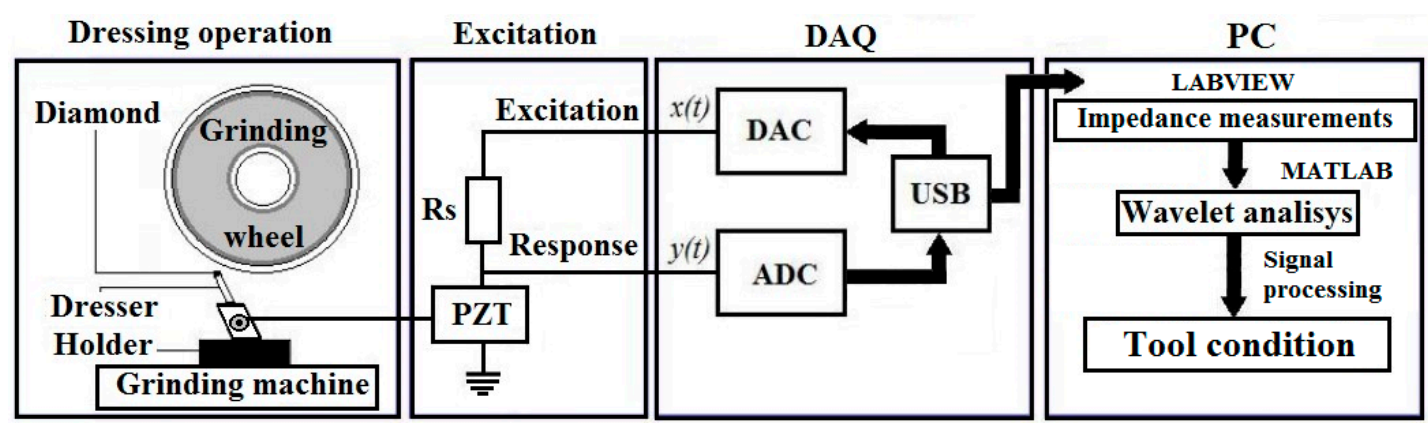

Figure 1. Proposed system configuration based on reference [9] using wavelet analysis for tool condition monitoring.

As a transducer, a low-cost PZT piezoelectric diaphragm, model 7BB-20-6, from MURATA Inc. (Smyrna, GA, USA), was used. This transducer consists of a circular brass of $20 \mathrm{~mm}$ diameter and $0.20 \mathrm{~mm}$ thickness, and a piezoelectric ceramic (active element) of $14 \mathrm{~mm}$ in diameter and $0.22 \mathrm{~mm}$ 
in thickness. The piezoelectric diaphragm was fixed to the dresser holder by a thin layer of cyanoacrylate glue. To monitor the temperature, a digital thermometer, model MT455 from MINIPA (São Paulo, Brazil), equipped with a k-type thermocouple was used. The reference temperature adopted for all measurements was $30^{\circ} \mathrm{C}$., The alternative EMI system proposed in reference by [11] was employed The DAQ device used in the present study was the NI USB-6221 model from National Instruments (Austin, Texas, EUA), with a sampling rate of $250 \mathrm{kS} / \mathrm{s}$ (kilo samples per second) to measure the impedance signatures. The transducer was excited by a $2.2 \mathrm{k} \Omega$ resistor and a $1 \mathrm{~V}$ amplitude chirp. According to Figure 1, the DAQ device is based on National Instruments LabVIEW ${ }^{\circledR}$ software, which has an analogical output to provide the output excitation signal $x(t)$ through the digital-to-analogical converter (DAC), as well as an analogical input for the signal response $y(t)$ acquisition via the analogical-to-digital converter (ADC). Subsequently, the digital content of the excitation and response signals, respectively, were processed on the computer. The connection between the DAQ device and the computer was achieved through a universal serial bus (USB). Timedomain voltage $(\mathrm{V})$ signal measurements corresponding to the electrical impedance signatures were recorded before the experiment (baseline) and during an interval of 100 dressing passes to detect structural damage. The digital processing of the collected signals was performed in the MATLAB ${ }^{\circledR}$ software.

The signal feature extraction was performed using the discrete wavelet transform. In this way, it is worth to note that, due to the multi-resolution capability of a wavelet transform, noisy signals can be separated into various approximation and detail signals by wavelet coefficients. Waveletbased damage diagnosis is made by comparing the approximation and detail coefficients for each wavelet level between baseline and damage condition signals $y[n]$ measured with the EMI method, as addressed by $[12,13]$. For this purpose, the MATLAB ${ }^{\circledR}$ wavedec function was implemented using the sym 4 wavelet family for decomposition, based on the approximation $c_{1}[k]$ and detail $d_{1}[k]$ coefficients computed from Equations (1) and (2), respectively.

$$
\begin{aligned}
& c_{1}[k]=\sum_{n} h_{1}[n-2 k] y[n] \\
& d_{1}[k]=\sum_{n} g_{1}[n-2 k] y[n]
\end{aligned}
$$

According to Equations (1) and (2), the wavelet decomposition is based on the pair of finite impulse response (FIR) digital filters, where the first level of decomposition is composed of a highpass filter and a low-pass filter with impulse responses $g_{1}[n]$ and $h_{1}[n]$, respectively, generating the approximation and detail coefficients. The next levels of approximation and detail are obtained by decomposing previous levels applying the impulse responses. Thus, the decomposition based on discrete wavelet transform consists of a maximum of $\log _{2} N$ stages, since each calculated level produces a half-reduced sample rate sequence $[12,13]$. In the present study, several levels of coefficients were tested by computing the correlation coefficient deviation metric (CCDM), using Equation (3), and the best levels were chosen to describe the condition of the dressing tool. Thus, this analysis will allow decision-making and estimation of the tool condition in the dressing operation, and consequently in the grinding process.

$$
C C D M=1-\left|\frac{\sum_{k}\left(C_{k, H}-\bar{C}_{H}\right)\left(C_{k, D}-\bar{C}_{D}\right)}{\sqrt{\sum_{k}\left(C_{k, H}-\bar{C}_{H}\right)^{2}} \sqrt{\sum_{k}\left(C_{k, D}-\bar{C}_{D}\right)^{2}}}\right|
$$

In Equation (3), $C_{k, H}$ represents the $k t h$ approximation or detail coefficient of the wavelet transform $(\mathrm{k}=1,2, \ldots \mathrm{N})$ in a healthy (baseline) condition and $C_{k, D}$ represents the $k t h$ approximation or detail coefficient of the wavelet transform for the damaged structure. This analysis is based on the methodology proposed in reference by [13], where the CCDM indices of the $C(k)$ coefficients were normalized from 0 to 1 . Thus, if the index tends to zero, there is no damage, and the lower values 
indicate lower residual variation. However, as the index increases, it assumes that damage has occurred to the structure, where the maximum value for the indices is $1(100 \%)$.

\section{Results and Discussions}

\subsection{Tool Wear Analysis}

Table 1 shows the worn area of the dresser diamond tip throughout the experiment, which was obtained from measurements taken sequentially at 100 passes (damage 1), 200 passes (damage 2), and 300 passes (damage 3). According to Table 1, the dresser diamond tip presented the most significant wear after 200 dressing passes, reaching the end of its useful life (i.e., when the diamond was completely damaged) at 300 dressing passes (100\% of wear). It is possible to observe that the diamond followed a trend throughout the experimental test, i.e., the worn area increases significantly to the number of passes.

Table 1. Wear area of the diamond.

\begin{tabular}{cccc}
\hline Dressing passes & 100 & 200 & 300 \\
\hline Tool wear (\%) & $28 \%$ & $55 \%$ & $100 \%$ \\
\hline
\end{tabular}

\subsection{Discrete Wavelet Analysis Based on EMI for Tool Condition Monitoring}

Normalized CCDM indices were obtained for the approximation and detail coefficients, which are shown at five wavelet decomposition levels in Figure 2. Thus, the conditions of the dressing tooli.e., baseline (healthy), 100 passes (damage 1), 200 passes (damage 2)m and 300 passes (damage 3)are shown at each of the decomposition wavelet levels. It is observed that the higher values of the CCDM indices are mainly concentrated in damage 3 (300 passes) for both approximation (Figure 2a) and detail (Figure $2 b$ ) coefficients.

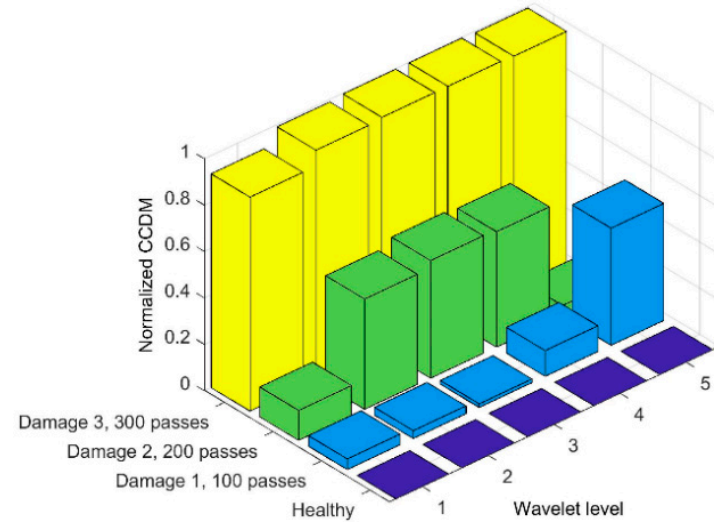

(a)

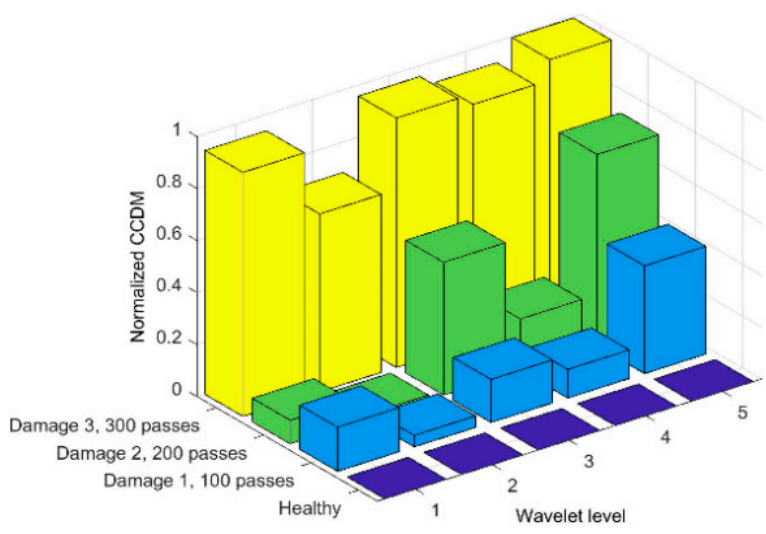

(b)

Figure 2. Normalized CCDM for (a) approximation and (b) detail coefficients.

Thus, as the dressing test was performed, the CCDM indices presented a trend of increasing in terms of amplitude. Therefore, the values were lower for damage 1 (100 passes). However, it can be observed that the trend at each particular level is not entirely linear and increasing. For example, the CCDM index for the approximation coefficients (Figure 2a) showed an utterly satisfactory behavior, in terms of correspondence with actual dressing tool condition, for practically all coefficient levels, except for level 5, where an increasing trend was not observed. On the other hand, according to Figure $2 \mathrm{~b}$, for the detail coefficients, most results of the CCDM indices were higher and more prominent when compared to the approximation coefficients (Figure 2a). For example, levels 3, $4 \mathrm{~m}$ and 5 showed entirely satisfactory results corresponding to the actual dressing tool condition, with particular focus to level 3, where the CCDM index presented the best result. 
However, for comparison purposes, level 4 presented the best performance between both approximation and detail coefficients, since levels 2 and 3 were not simultaneously satisfactory for both cases. For further details, an enlargement is shown in Figure 3, where a comparison of the approximation and detail coefficients of the time domain response signals corresponding to baseline (healthy) and 300 dressing passes (damage 3), respectively, were made. Thereby, from an enlargement of the graph, it is possible to note variations between baseline and damage condition, characterizing the level 4 sensitivity for both approximation and detail coefficients to estimate the dressing tool condition.

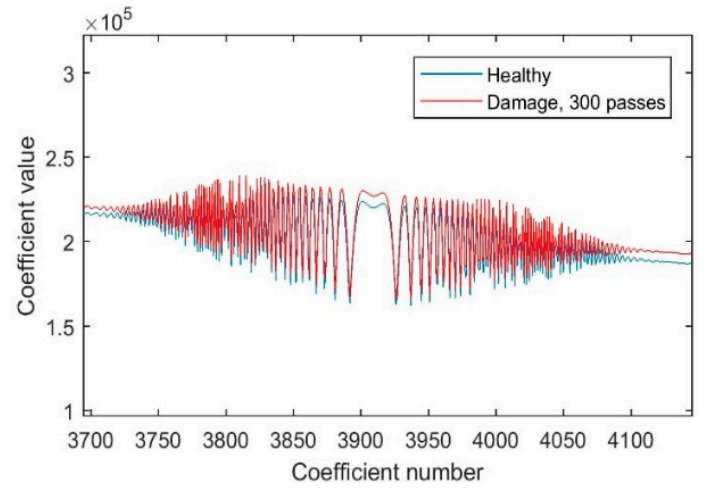

(a)

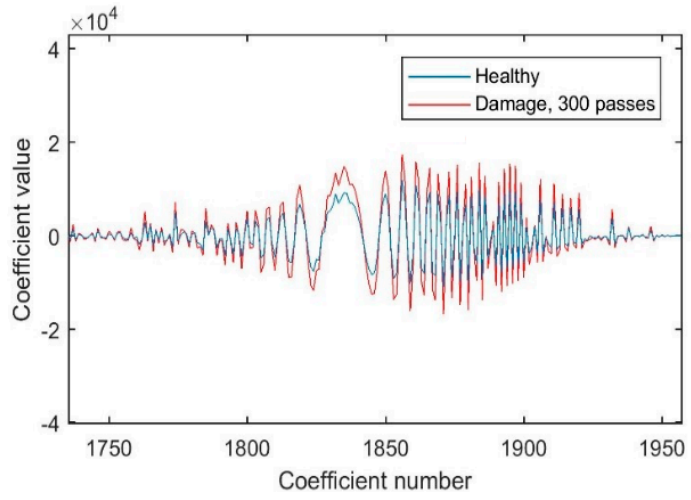

(b)

Figure 3. Magnification for (a) approximation and (b) detail coefficients at level 4.

\section{Conclusions}

This work aimed to contribute to the condition monitoring of the dressing tool based on the EMI method using wavelet analysis, in which the approximation and detail coefficients estimated the tool condition. By selecting an appropriate decomposition level for the approximation and detail coefficients, it was possible to identify a correspondence with the actual dressing tool condition (wear level) using the CCDM metric. In this context, as the damage occurs in the diamond, the indices become more prominent, with particular focus to level 4, which presented the best correspondence with the dressing tool condition in both approximation and detail coefficients. From this analysis, an amplitude threshold can be set before the dresser diamond tip is damaged, avoiding dressing operations performed with damaged tools, thus providing the correct overlap ratio and sharpness to the grinding wheel.

Author Contributions: P.A. conceived and designed the experiments; P.O.J. performed the experiments; F.B. detected the sensor signals; S.C. and R.R. developed the data processing procedures; F.A., C.S.J., and M.V. overviewed the whole work and supported the discussion of the results.

Funding: This research was funded by the São Paulo Research Foundation, grant \#2016/02831-5.

Acknowledgments: The authors are grateful to the anonymous reviewers for improving this paper.

Conflicts of Interest: The authors declare no conflict of interest.

\section{References}

1. Pombo, I.; Cearsolo, X.; Sánchez, J.A.; Cabanes, I. Experimental and numerical analysis of thermal phenomena in the wear of single point diamond dressing tools. J. Manuf. Process. 2017, 27, 145-157.

2. Alexandre, F.A.; Lopes, W.N.; Dotto, F.R.L.; Ferreira, F.I.; Aguiar, P.R.; Bianchi, E.C.; Lopes, J.C.; Aguiar, P.R. Tool condition monitoring of aluminum oxide grinding wheel using AE and fuzzy model. Int. J. Adv. Manuf. Technol. 2018, 96, 67-79.

3. Alexandre, F.; de Aguiar, P.; Götz, R.; Aulestia Viera, M.; Lopes, T.; D’addona, D.; Bianchi, E.; da Silva, R.B. Emitter-Receiver Piezoelectric Transducers Applied in Monitoring Material Removal of Workpiece during Grinding Process. Proceedings 2019, 4, 9. 
4. Oliveira Junior, P..; Ferreira, F.I.; Aguiar, P.R.; Batista, F.G.; Bianchi, E.C.; Daddona, D.M. Time-domain Analysis Based on the Electromechanical Impedance Method for Monitoring of the Dressing Operation. Procedia CIRP 2018, 67, 319-324.

5. Martins, C.H.R.; Aguiar, P.R.; Frech, A.; Bianchi, E.C. Tool Condition Monitoring of Single-Point Dresser Using Acoustic Emission and Neural Networks Models. IEEE Trans. Instrum. Meas. 2014, 63, 667-679.

6. Miranda, H.I.; Rocha, C.A.; Oliveira Junior, P.; Martins, C.; Aguiar, P.R.; Bianchi, E.C. Monitoring singlepoint dressers using fuzzy models. Procedia CIRP 2015, 33, 281-286.

7. Oliveira Junior, P.; Marchi, M.; Martins, C.H.R.; D'Addona, D.; de Aguiar, P.R.; Bianchi, E.C. Spectral estimation of vibration signal for monitoring the wear of single-point dresser. Rev. Mater. 2016, 21, 827-840.

8. D'Addona, D.M.A.; Lopes, S.C.W.N.; Aguiar, P.R.; Bianchi, E.C.; Teti, R. Tool condition monitoring of single-point dressing operation by digital signal processing of AE and AI. Procedia CIRP 2018, 67, 307-312.

9. Junior, P.; D'Addona, D.M.; Aguiar, P.R. Dressing Tool Condition Monitoring through Impedance-Based Sensors: Part 1-PZT Diaphragm Transducer Response and EMI Sensing Technique. Sensors 2018, 18, 4455.

10. Oliveira Junior, P.; D’Addona, D.; Aguiar, P.; Teti, R. Dressing Tool Condition Monitoring through Impedance-Based Sensors: Part 2-Neural Networks and K-Nearest Neighbor Classifier Approach. Sensors 2018, 18, 4453.

11. Baptista, F.G.; Vieira, J.F. A new impedance measurement system for PZT based structural health monitoring. IEEE Trans. Instrum. Meas. 2009, 58, 3602-3608.

12. Castro, B.A.; Baptista, F.G.; Ciampa, F. Comparative analysis of signal processing techniques for impedance-based SHM applications in noisy environments. Mech. Syst. Signal Process. 2019, 126, 326-340.

13. Inman, D.J.; Vieira, J.; Guimar, F. Time-domain analysis of piezoelectric impedance-based structural health monitoring using multilevel wavelet decomposition. Mach. Syst. Signal Process. 2011, 25, 1550-1558.

14. Grabowska, J.; Palacz, M.; Krawczuk, M. Damage identification by wavelet analysis. Mech. Syst. Signal Process. 2008, 22, 1623-1635.

15. Oliveira Junior, P.; Souza Ruzzi, R.; Lopes, W.N.; Alexandre, F.A.; Baptista, F.G.; de Aguiar, P.R.; Bianchi, E.C. A New Approach For Dressing Operation Monitoring Using Voltage Signals Via Impedance-Based Structural Health Monitoring. KnE Eng. 2018, 942-952.

16. Oliveira Junior, P.; Conte, S.; D’Addona, D.M.; Aguiar, P.R.; Baptista, F.G.; Bianchi, E.C.; Teti, R.. Damage patterns recognition in dressing tools using PZT-based SHM and MLP networks. Procedia CIRP 2019, 79, 303-307.

(C) 2019 by the authors. Licensee MDPI, Basel, Switzerland. This article is an open access article distributed under the terms and conditions of the Creative Commons Attribution (CC BY) license (http://creativecommons.org/licenses/by/4.0/). 